\title{
Préparation de particules monodispersées de cobalt et de nickel de taille micronique et submicronique
}

\author{
F. FIEVET, F. FIEVET-VINCENT, J.-P. LAGIER, B. BEAUDOIN* et M. FIGLARZ*
}

Laboratoire de Chimie des Matériaux Divises et Catalyse, Universite Paris 7, 2 Place Jussieu, 75251 Paris sedex 05, France

Laboratoire de Réactivité et de Chimie des Solides, Universite de Picardie, 33 rue Saint Leu, 30039 Amiens cedex, France

Des particules de cobalt ou de nickel quasi sphériques, présentant une faible dispersion de taille sont obtenues par réduction d'un précurseur inorganique par un polyol liquide. La réaction a lieu en solution, les particules métalliques se formant par germination et croissance à partir de la phase liquide.

Lorsque la nucléation est spontannée (homogène) les paramètres expérimentaux tels que la température, la composition du milieu et en particulier la nature et la concentration du précurseur permettent de faire varier le diamètre des particules de 1 à $4 \mu \mathrm{m}$.

Par ensemencement du milieu par des germes métalliques (nucléation hétérogène) on peut réduire la taille des particules jusqu'à quelques dixièmes de micromètres et la contrôler en jouant sur la quantité de germes introduits.

On peut également modifier nettement la forme des particules en les précipitant en présence d'un champ magnétique.

Les différents types de poudres sont décrits, leur aimantation à saturation et leur champ coercitif ont été mesurés.

\section{REFERENCES}

1- F. FIEVET, J.P. LAGIER et M. FIGLARZ, M.R.S. Bulletin, 14 (1989), p 29.

2- F. FIEVET, J-P. LAGIER, B. BEAUDOIN, B. BLIN et M. FIGLARZ, Solid State Ionics 32/33 (1989) p 198. 